\title{
A Novel Optimizing Mechanism of Load Balancing in LTE Network
}

\author{
Luan $\mathrm{Yu}$ \\ Research Center \\ China Railway Communication Co., Ltd. \\ Beijing, China \\ 15011579400@139.com
}

\author{
Wang Bo, Lv Zhaobiao, Wang Jianquan \\ Wireless Lab, Network Technology Research Center \\ China Unicom Research Institute \\ Beijing, China \\ Wangbo149@chinaunicom.cn
}

\begin{abstract}
With the rapid developing of wireless communication technology and the growing user service, the demand on the system capacity from users is growing. In the LTE network, the uneven load distribution makes that network resource is not fully utilized, which results that the capacity is limited. In this paper, a novel optimizing mechanism of network load balancing based on the network manager is proposed. The eNBs in the network will broadcast the current load status by the way of adapting a new message format with the life cycle bit when the load of the cell is changed, then the serving eNB can decide which part of users in the cell should transfer to the neighboring cells and send a request to the network manager. The network manager will calculate whether the transferred users make the neighboring cell load exceed the threshold. If not, the users can be transferred to the neighboring cell and release the serving cell's load burdon. Otherwise, the serving cell will search other neighboring cells to transfer the users. The simulation results show that the proposed mechanism will not consume more network resource and improve the network throughput significantly.
\end{abstract}

Keywords-MLB; Load Balancing; network manager; Ping Pong Effect; Self-Optimizing

\section{INTRODUCTION}

Recently, the wireless communication market rise rapidly due to the development of wireless communication technologies. Modern wireless networks are able to support multiple high data rate multimedia services, such as voice, data, and video simultaneously. Along with exploding users' demand of high data rate multimedia wireless services, there are much more requirements on the capacity of wireless network. Thereby, how to optimize network loads become much more important to telecom operators.

Each operator can have various wireless networks simultaneously, such as GSM, WCDMA and WLAN, which results that more networks can coexist. As the result, the network parameters which need to be tuned are increasing exponentially, and the OPEX goes up rapidly as well. In order to reduce the operational cost, the network need to introduce self-optimizing network technologies, which can apperceive the changes of wireless network environment and then adjust the network parameters automatically, eventually improve the whole network performance.
Mobility Load Balancing (MLB) is one of the important features in the self-optimizing technology [1,2]. MLB can move the users from heavy load cell to the neighboring light load cells by adjusting handover region. In MLB scenarios, load may be transferred between two cells frequently. This situation is so called ping pong effect. This ping pong effect consumes limited network recourses and impacts the continuity of services.

According to the principle of Greedy algorithm, Professor H.L. Hou and J. Zhang proposed a Mobility Load Balancing scheme [3], which is based upon transmission delay and average handover time. This MLB scheme can improve 20 percent of the whole system capacity, but still cannot figure out the problem of ping pong effect. To resolve the ping pong effect, a multi-hop MLB scheme was proposed [4]. However this multi-hop MLB scheme didn't have strict definition for network convergence conditions, whcih induces a mass of control signals and reduces the whole network capacity.

In this paper, a novel optimizing mechanism of network load balancing based on the network manager is proposed, which aims to fix the ping-pang effect. The serving eNB receives the current load value and load threshold from the neighboring cells, then calculate which part of users in the serving cell to be transferred and select the amount of transferred load. Then, the serving eNB add the transferred load with the current load value of an adjacent cell. If the sum is smaller than the load threshold of the adjacent cell, the neighboring cell is a candidate cell for the transferred load. The serving eNB will report the cell identification of the candidate cell, the current load value of the candidate cell and the amount of transferred load to the network manager. The network manager calculates the total amount load transferred to the same candidate cell and decides if the transferred load with current load value beyond the threshold. When it is less than the candidate cell load threshold, the candidate cell can be a target cell of serving cell. Then, the serving cell transfers the users to the target cell and releases the load burden. What's more, the proposed mechanism is verified on the LTE system simulation platform. The simulation results show that the proposed mechanism will not consume more network resource and improve the network throughput.

The remainder of this paper is structured as follows: In section II, the ping pong effect of load transfer in the MLB is 


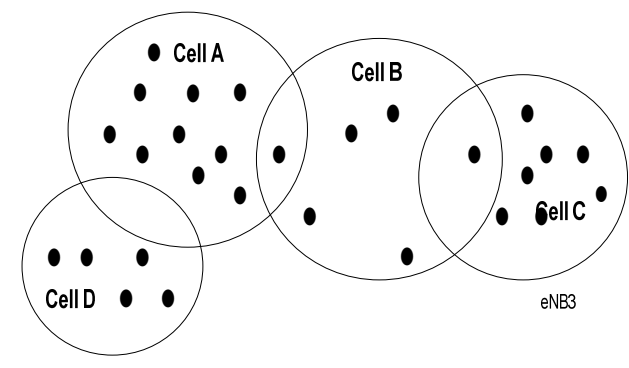

Figure 1. The scenario of load transfer ping pong effect.

descripted in detail. The proposed optimizing mechanism based on the network manager is discussed in section III. Section IV verifies the proposed optimizing method and analyzes the test result data. Finally, we conclude this paper in Section V.

\section{PingPang EFFECT OF LOAD TRANSFER}

In LTE system, the situation of overload usually occurs among some cells. For example, some users served by the same cell watching the video at the same time. Then they will occupy the whole system bandwidth which result that other service can not be meet and make the network quality degradation. At the first stage of LTE deployment, LTE network, which is used as a complementary technology of 3G network, should be deployed in some hot spots such as business district or shopping mall. When the cell in these scenarios is overload, it will not only impact the cell itself, but also interference the neighboring cells. So it's reasonable to use load balancing in these scenarios. 3GPP has brought forward a few solutions for that problem after a deep discussion [5]. However, the solution only considers the load of serving cell and neighboring cells, before optimizing the handover parameters, which is not enough accurate. When two overload cells move users to the same light load cell, the phenomenon of load ping pong transfer will appear. This load ping pong transfer situation wastes a mass of bandwidth recourse. The detail description is in R3-101477 [6], as shown in the Figure 1.

In Figure 1, cell A is a heavy load cell, so it has to move part of its users to neighboring cell. After negotiating with cell $\mathrm{B}$ and Cell D, cell A decide to move part of its users to cell B. At the moment, cell $\mathrm{C}$ is overload as well, it also needs to transfer its users to cell $\mathrm{B}$. There are several ways to deal with this situation in the following:

- Cell B rejects cell C's requests. Since cell B already accept some users from cell $\mathrm{A}$ and there no more resources for users from cell $\mathrm{C}$.

- Cell B may accept part of users in cell C. For example, the spare capacity of cell $B$ is 80 users. After receiving 50 users from cell $\mathrm{A}$, there is still 30 users' resources left. But in practice, cell $\mathrm{C}$ needs to

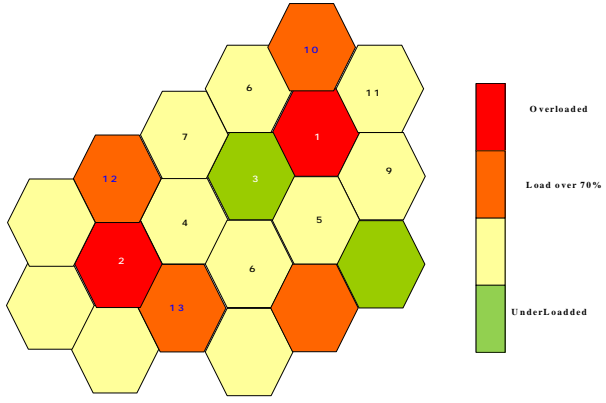

Figure 2. The load transfer ping pong effect.

move 40 users to cell B. In this situation, cell B can only accepts parts of users from cell C.

- There are no enough resources in cell B, so cell B request to transfer parts of its users to cell $A$ and make room for cell C's loads. But after receiving loads from cell $\mathrm{B}$, cell $\mathrm{A}$ will be overload. At the moment, MLB works in cell A and start to transfer users to cell B again, then cell B transfer loads to cell $\mathrm{C}$, cell $\mathrm{C}$ will be overload as well and transfer users to cell B. This situation which is also called load ping pong effect, make the network resource utilization wasteful.

In order to solve the above problems, there are three solutions in the following $[7,8]$.

- Solution A: Before startup of MLB, the overload cell should know the load state of its neighboring cells and the neighbor's neighboring cells, then decide where to move its users according to these information.

- Solution B: The overload cell chooses several candidate cells from its neighboring cells, then start MLB according to the load situation of these candidates.

- Solution C: The overload cell regards all neighbor cells as its load balancing candidate cells, then it tries to choose the cell one by one, until finding the appropriate target cell. The drawback of this solution is that when all the neighbor cells don't satisfy the load balancing condition, load balancing will not be executed.

\section{The OptIMIZING MECHANISM}

Although the three solutions mentioned above can solve some problems, but also bring a lot of signaling load. Before the cells start to load balancing, the signaling message is the main exchange information with neighboring cells and their neighbors. However, this will cause a sudden increase of load information signaling, which is not conducive to the stability of the network. Analysis of the scene is shown in Figure 2: 


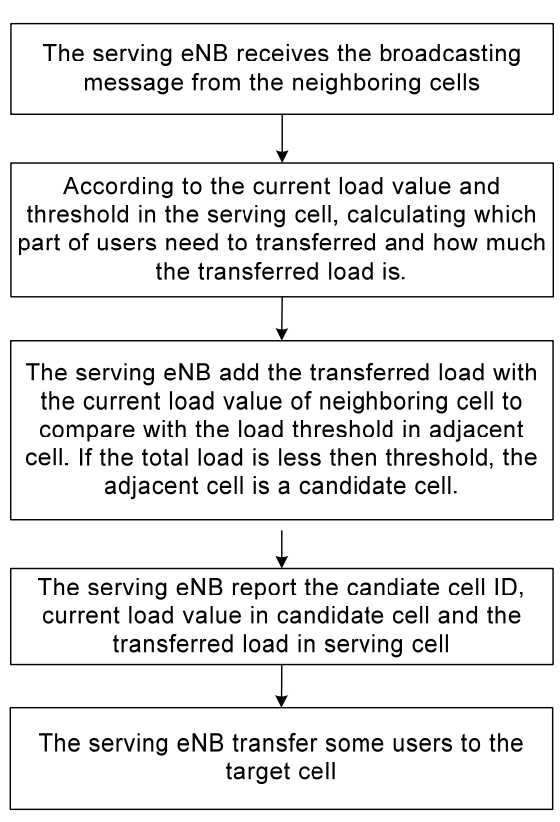

Figure 3. The process of proposed mechanism.

In Figure 2, when Cell 1 is overload, it will send a message to the neighboring cells and neighbor's neighboring cells to require their load states. According to the load distribution in Figure 2, Cell 1 will choose Cell 3 as its target cell. So, the Cell 1 moves some users to the Cell 3, and Cell 1 will be light load. At the same time, Cell 2, Cell 12 and Cell 13 also move some users to the Cell 4 . However, this will make the Cell 4 be too heavy load district, so the Cell 4 will move some users to the Cell 3 which makes the Cell 3 is also too heavy. Then Cell 3 will move its own users to the Cell 1. This back and forth to carry out the MLB action, results in a waste of resources.

To solve these problems, this paper proposes a novel optimizing mechanism of network load balancing based on the network manager. The eNBs in the network will broadcast the current load status when the load of the cell is changed, then the serving eNB can decide which part of users in the cell should transfer to the neighboring cells and send a request to the network manager. The network manager will calculate whether the transferred users make the neighboring cell load exceed the threshold. If not, the users can be transferred to the neighboring cell and release the serving cell's load burdon. Otherwise, the serving cell will search other neighboring cells to transfer the users. The process of proposed mechanism is shown in figure 3. The detail steps are as following:

Step1: the serving eNB receives the broadcasting messages from the neighboring cells. In this step, the message includes the sending neighbor cell's current load value and load threshold.

Step2: According to the current load value and threshold in the serving cell, calculating which part of users need to transferred and how much the transferred load is.

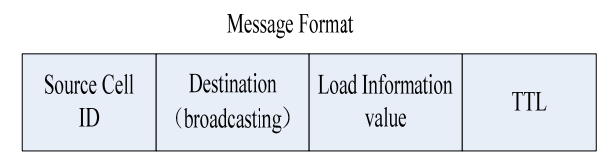

Figure 4. The design of message format.

Step3: The serving eNB add the transferred load with the current load value of neighboring cell to compare with the load threshold in adjacent cell. If the total load is less then threshold, the adjacent cell is a candidate cell.

Step4: The serving eNB report the candidate cell ID, current load value in candidate cell and the transferred load in serving cell. The network manager calculates the total amount load transferred to the same candidate cell and decides if the transferred load with current load value beyond the threshold. When it is less than the candidate cell load threshold, the candidate cell can be a target cell of serving cell.

Step5: The serving eNB transfer some users to the target cell.

In the proposed mechanism, the broadcasting message format is designed in figure 4 . The most field in the message format is the TTL(Time to Live) which is made to avoid signaling overload. When the broadcasting message is forward one time, the value of TTL is reduced by 1 . If the TTL value is 0 , the message will be discarded.

The proposed optimizing mechanism is based on the network manager to make the decision, which takes into account not only the information of the neighbor's neighbor cells, also takes into account the global information network. So each eNB can select the candidate cell as target cell properly, and not cause the load ping pong effect occurred in the scenario of MLB. What's more, the proposed mechanism is apt to put into use in the operator's network.

\section{VERIFICATION}

In order to verify the proposed optimizing mechanism, the dynamic simulation system is adopted [9]. The functionalities implemented include channel model, mobility, handover, and power control. These simulations have been conducted in LTE scenario which is composed of seven eNBs and several users. The most relevant simulation parameters have been collected to Table I. In the simulation environment, there is a total of seven base stations which controls three sectors.

TABLE I. THE PARAMETERS OF SIMULATION

\begin{tabular}{|l|l|}
\hline \multicolumn{1}{|c|}{ Parameter } & \multicolumn{1}{c|}{ Assumption } \\
\hline 3GPP Macro Cell Scenario & Cell layout 57 sectors \\
\hline Antenna pattern & $\begin{array}{l}\text { 70-degree sectored } \\
\text { beam }\end{array}$ \\
\hline Distance-dependent path loss & $128.1+37.6 l o g(r)$ \\
\hline Shadowing standard deviation & $8 \mathrm{~dB}$ \\
\hline UE ditstribution & Uniform \\
\hline Traffic model & Full buffer \\
\hline Duplexing & FDD \\
\hline
\end{tabular}




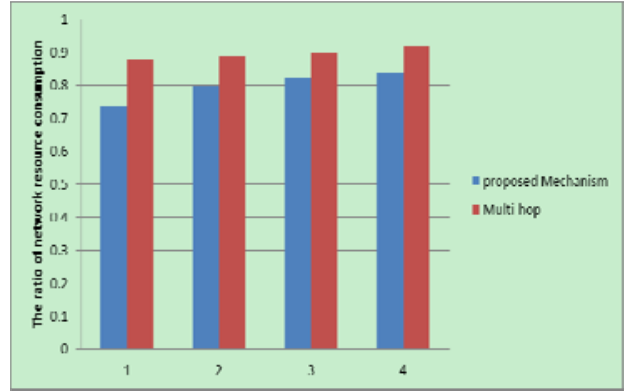

Figure 5. The network resource consumption.

Figure 5 illustrates that the proposed optimizing mechanism can achieve load balancing and fast convergence. Compared with the multi-hop MLB algorithm, the proposed algorithm occupies less network resources. The main reason is that the proposed algorithm using the centralized architecture among which the network manager can get the whole network information, can make the network fast convergence. What's more, a new broadcasting message format is adopted without causing signaling flooding to avoid consuming more network resources.

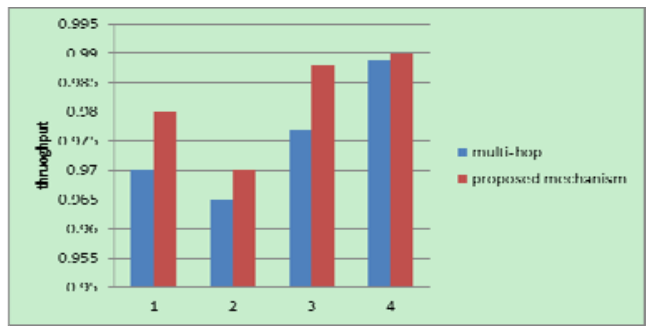

Figure 6. The network throughput.

Figure 6 shows that the proposed optimizing algorithm can significantly improve overall network throughput. Compared to multi-hop MLB algorithm which achieves load balancing step by step, taking into account only the neighboring cells' load information around the overload cell, the proposed optimizing algorithm considers the load of the entire network on the network manager platform, so it can significantly improve the overall network throughput.

\section{CONCLUSION}

This paper analyzes the load transfer ping pong effect within the mobility load balancing in LTE network, which waste a mass of network resource. In order to solve the problem, a novel optimizing mechanism of network load balancing based on network manager is proposed. The optimizing mechanism is a centralized-control method which takes into account not only the information of the neighbor's neighbor cells, also takes into account the global information network. So each eNB can select the appropriate target cell. What's more, the simulation results show that the proposed optimizing mechanism using a special broadcasting message format, can make the network fast convergence without causing signaling flooding, and significantly improve overall network throughput.

\section{REFERENCES}

[1] N. Timo; T. Jussi, V. Ingo, "Performance of LTE self-optimizing networks uplink load balancing”, in the Proc. 2011 IEEE 73rd Vehicular Technology Conference, VTC2011-Spring, 2011.

[2] Ma Dong, Ma Maode, "Proactive load balancing with admission control for heterogeneous overlay networks", Wireless Communications and Mobile Computing, 2011.

[3] Honglin, Hu., Jian Z., Xiaoying Z., Yang Y., Ping W. , SelfConfiguration and Self-Optimization for LTE Net-works, IEEE Communications Magazine, 2010.

[4] Liu Zhiqiang, "The study of mobility load balancing in the LTE netowrk”, University of Science and Technology of China, 2011.

[5] 3GPP TS 36.331, Evolved Universal Terrestrial Radio Access (EUTRA); Radio Resource Control (RRC); Protocol specification (S), 2010

[6] 3GPP R3-101477, Requirement for further enhancement of MLB (S/R), CMCC, RAN3\#68.

[7] 3GPP R3-102107, An enhancement for MLB (S/R), CMCC, RAN3\#69.

[8] 3GPP R3-102763, Discussion on Further MLB Enhancement (S/R), CMCC, RAN3\#69bis.

[9] J. C. Ikuno, M. Wrulich, M. Rupp, “System level simulation of LTE networks", in the Proc. 2010 IEEE 71st Vehicular Technology Conference(C), Taipei, Taiwan, May 2010. 